\title{
Sjögren's syndrome and lymphadenopathy unraveling the diagnosis of Lyme disease
}

\author{
Svitlana Smiyan ${ }^{1}$, Igor Galaychuk ${ }^{2}$, Igor Zhulkevych², Volodymyr Nykolyuk ${ }^{3}$, Roman Komorovsky², \\ Sofiya Gusak ${ }^{1}$, Ivan Bilozetsky ${ }^{4}$ \\ ${ }^{1} 2^{\text {nd }}$ Department of Internal Medicine, Ivan Horbachevsky Ternopil State Medical University, Ukraine \\ ${ }^{2}$ Department of Oncology and Radiology, Ivan Horbachevsky Ternopil State Medical University, Ukraine \\ ${ }^{3}$ Department of Pathohistology, Ternopil Regional Oncology Center, Ukraine \\ ${ }^{4}$ Department of Surgical Dentistry, Ivan Horbachevsky Ternopil State Medical University, Ukraine
}

\begin{abstract}
Lyme disease is a multisystem tick-borne transmissive disease with heterogeneous manifestations, frequently making the diagnosis difficult in clinical practice. Herein, we report a case of a 43-year-old female patient who presented with generalized lymphadenopathy requiring a further diagnostic evaluation towards lymphoma. The patient also had clinical signs of Sjögren's syndrome. Lymph node excision with subsequent histological and immunohistochemical studies were performed. While light microscopy findings were suspicious for lymphoma, immunohistochemistry results were consistent with cortical and paracortical lymph node hyperplasia with no evidence of lymphoproliferative disorder. Further laboratory testing confirmed the presence of Lyme borreliosis. Effectiveness of the administered antibiotic therapy confirmed Borrelia burgdorferi infection. Interrelationships between Sjögren's syndrome, lymphadenopathy and Lyme disease are discussed.
\end{abstract}

Key words: Sjögren's syndrome, lymphadenopathy, Borrelia burgdorferi, Lyme disease.

\section{Introduction}

Lyme disease is a multisystem disorder which is frequently called a "great imitator" because of the diversity of its clinical manifestations. This is a complicated disease of infectious etiology which in its expression is inflammatory or rheumatic [1, 2], and thus it may serve as a unique human model for the study of rheumatic diseases of infectious etiology [3, 4].

Lyme borreliosis is the most prevalent transmissive tick-borne disease in the northern hemisphere, accounting for $>90 \%$ of all tick-borne diseases with an annual incidence of 300,000 cases in the United States [5] and 65,000 cases in Europe [1]. The disease is characterized by a chronic course with predominant involvement of skin, heart, lymph nodes, musculoskeletal and nervous system, and spring and autumn incidence peaks related to seasonality of tick activity. The onset of the disease is commonly associated with systemic manifestations, such as fever, myalgias, arthralgias, and fatigue, and may be followed by systemic autoimmune joint diseases, including rheumatoid arthritis, psoriatic arthritis, and peripheral spondyloarthropathy $[3,4,6,7]$.

\section{Case report}

A 43-year-old woman was referred to the rheumatology clinic with complaints of a low-grade fever, generalized swelling of lymph nodes, migrating aches in joints of arms and legs, dryness in mouth and eyes, weight loss, and fatigue for the past 6 months. Her referral was preceded by evaluation for lymphadenopathy in the oncology clinic, including computed tomography (CT) scan (Fig. 1) and histological analysis of the excised left axillary lymph node (Fig. 2).

Histological examination revealed lymphatic node tissue with altered general architecture due to presence of lymphoid cells of variable size and irregular shape 


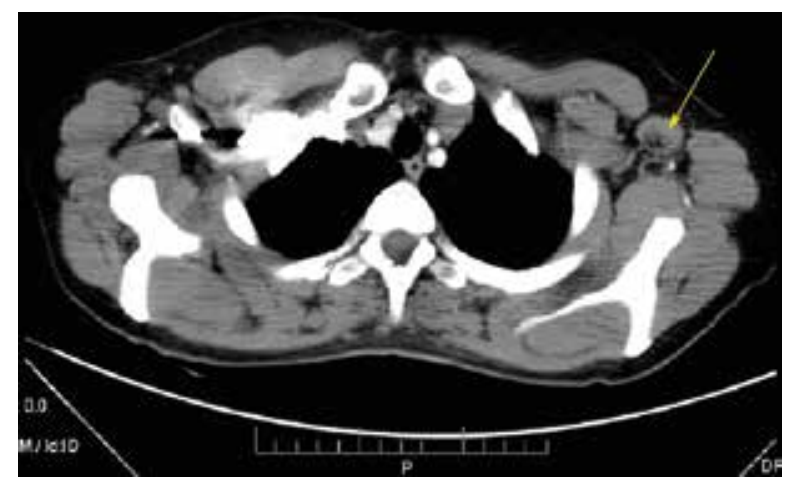

Fig. 1. Axial chest contrast-enhanced CT image showing the largest detected axillary lymph node measuring $4 \mathrm{~cm}$ in its greatest dimension (arrow). arranged in follicular groups with or without proliferation centers (Fig. 3). These findings were suspicious for follicular lymphoma, necessitating confirmation by immunohistochemical analysis.

Immunohistochemistry showed:

- CD3 (Dako, polyclonal) - positive reaction of small lymphocytes of paracortical zone and of rare lymphocytes of the cortical zone, including germinal centers.

- CD20 (Dako, Clone L26) - positive reaction of small lymphocytes of the cortical zone and of cells of the germinal centers as well as of rare lymphocytes of the paracortical zone.
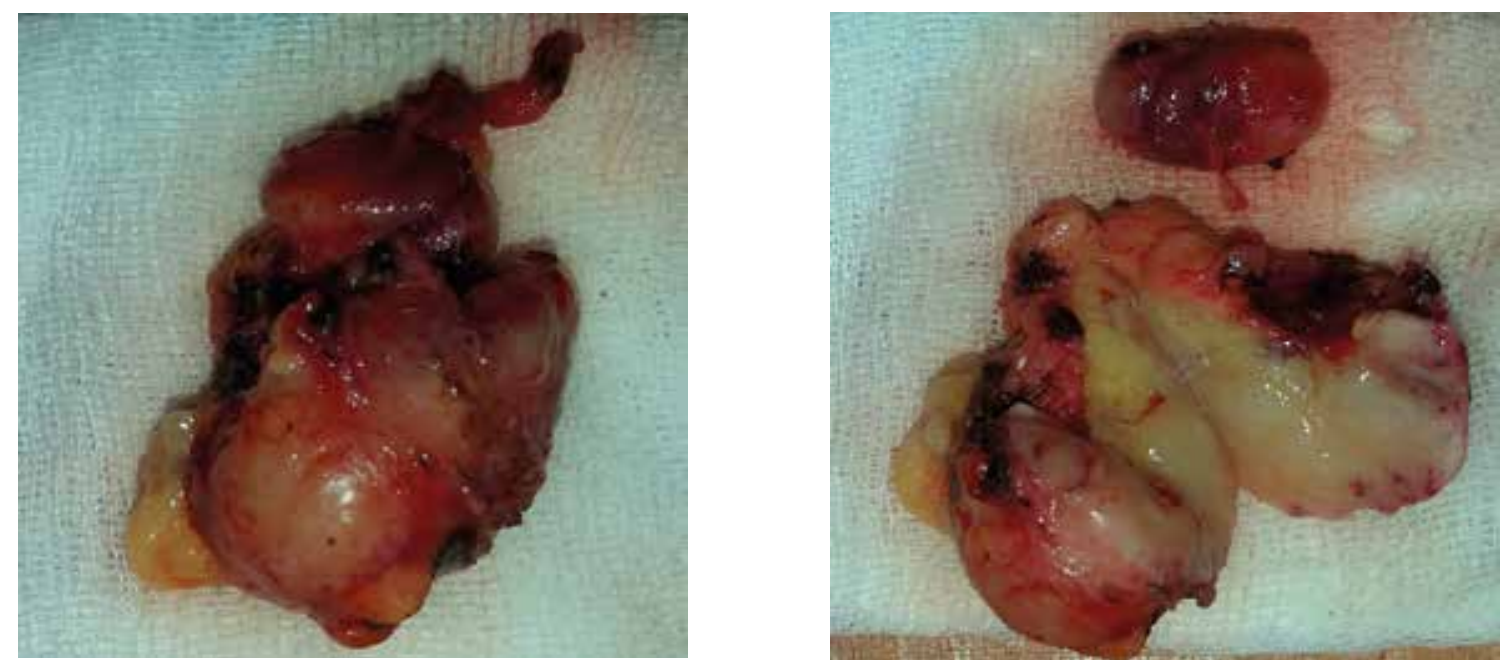

Fig. 2. Surgical specimen of the excised left axillary lymph node conglomerate $4.5 \mathrm{~cm} \times 4.0 \mathrm{~cm} \times 3.5 \mathrm{~cm}$ (left), and yellow-grayish cut surface of the same lymph nodes (right).



Fig. 3. Light microscopy appearance of an excised lymph node transverse section showing altered general architecture due to presence of lymphoid cells of variable size and irregular shape arranged in follicle-like groups with lymphoid proliferation centers (arrowheads) and pseudogranulomas (arrows) (hematoxylin-eosin staining, $100 \times$ ).

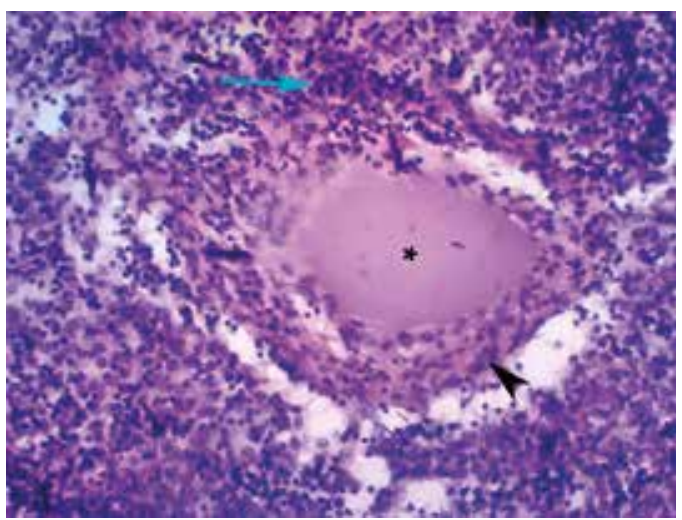

Fig. 4. Histopathologic findings in a lymph node consistent with specific granulomatous inflammation: pseudogranuloma (asterisk) with homogeneous structure surrounded by lymphohistiocytic infiltration (arrow) and a forming connective tissue pseudocapsule (arrowhead) (hematoxylin-eosin staining, $200 \times$ ). 
- Bcl-2 (Dako, Clone 124) - positive reaction of lymphocytes of the mantle, marginal and paracortical zone and of rare lymphocytes in the germinal centers; negative reaction in most of the lymphocytes in the germinal centers.

- CD30 (Dako, Clone Ber-H2) - positive reaction in rare cells with immunoblast morphology found in germinal centers and paracortical zone.

- Ki-67 (Dako, Clone MIB-1) - positive reaction in almost $100 \%$ of cells of secondary follicle germinal centers, positive reaction in rare cells of the paracortical zone.

- Human immunodeficiency virus capsid p24 protein (Dako, Clone Kal-1) - negative reaction.

Overall, the pathomorphological study showed preserved nodal cellular structure, almost all cells of the germinal centers were positive for Ki-67 proliferation marker, and most of the cells were represented by $B$ lymphocytes (CD20+, Bcl-2-) admixed with rare T lymphocytes (CD3+, Bcl-2+). In paracortical zones the cells were represented predominantly by $\mathrm{T}$ lymphocytes (CD3+, $\mathrm{BCl}-2+$ ) with increased proliferative activity and rare $B$ lymphocytes (CD20+, Bcl-2+). No signs of cytological atypia were detected in cells of cortical and paracortical zones. Staining for CD30 was positive only in immunoblasts. Therefore, no evidence of malignant lymphoproliferative disease was found. Lymph node alterations were interpreted as mixed (cortical and paracortical) hyperplasia.

Upon detailed history taking, it was revealed that the patient suffered a tick bite a year ago. No rash was seen on inspection. Physical examination revealed signs of lymphadenopathy: there were enlarged palpable cervical lymph nodes up to $1.5 \mathrm{~cm}$ in size, submandibular, right axillary lymph nodes up to $2 \mathrm{~cm}$ in size, elastic, movable, painless to mildly tender. Complete blood count and blood chemistry were normal.

An ophthalmologist and a dentist concluded that the patient had keratoconjunctivitis sicca and xerostomia. Further testing detected a significant decrease of the unstimulated whole saliva flow rate to $0.06 \mathrm{ml} / \mathrm{min}$, and Schirmer's test was $2 \mathrm{~mm} / 5 \mathrm{~min}$. The patient was also tested for serum presence of autoantibodies and positivity of antinuclear antibodies, anti-Ro (SSA), anti-La (SSB), anti-SS-A 52/60 lgG, and anti-SS-B IgG antibodies was demonstrated.

Double-stranded DNA antibodies, anti-ribosomal $P$ protein antibodies, anticentromere antibodies, Sm, Sm/RNP, RNP, Scl-70, and Jo-1 antibodies were not found. These results allowed for the diagnosis of Sjögren's syndrome (> 4 points) according to the current criteria [6], without biopsy of the minor salivary glands.

Considering the history of a tick bite, the patient underwent serological testing for Lyme borreliosis. Enzyme immunoassay and Western blot analysis were positive for specific anti-Borrelia burgdorferi IgG and IgM and negative for Borrelia afzelii and Borrelia garinii antibodies.

In the light of these findings, the histological lymph node specimens were reassessed. Attention was drawn by pseudogranulomas with homogeneous contents surrounded by lymphohistiocytic infiltration (Fig. 4) and a forming connective tissue pseudocapsule containing numerous macrophages (Fig. 5) indicative of specific granulomatous inflammation in lymph nodes.

Therefore, histology and immunohistochemistry data (CD20, Ki-67) finally were concluded to represent specific lymph node hyperplasia in response to Borrelia spirochete invasion. This lymph node hyperplasia was initially erroneously presumed to be a manifestation of a malignant lymphoma.

On the basis of the above findings, the patient was also diagnosed with Lyme borreliosis associated with Sjögren's syndrome.

Oral doxycycline $200 \mathrm{mg} /$ day was administered for 30 days, resulting in improvement of the patient's symptoms: the body temperature normalized, and oral and ocular sicca symptoms decreased. Also, swollen cervical lymph nodes returned to normal, and the size of axillary lymph nodes significantly decreased.

\section{Discussion}

This clinical case illustrates presentation of Lyme disease as Sjögren's syndrome and lymphadenopathy. Our findings support a previously published experimental study [7] in which similar immunohistochemistry findings were observed in lymph nodes during Lyme borreliosis: the authors demonstrated that Borrelia-specific $B$ cell responses are strongly induced in regional



Fig. 5. Fragment of the same tissue shown in Figure 4 demonstrating part of a pseudogranuloma (asterisk), a pseudocapsule (arrows), lymphocytes (white arrowhead), and macrophages (black arrowhead) (hematoxylin-eosin staining, $400 \times$ ). 
lymph nodes following infection, permitting the authors to conclude that lymphadenopathy during $B$. burgdorferi infection is caused by the accumulation of viable spirochetes in lymph nodes. Arvikar et al. [8] stated that other autoimmune diseases such as rheumatoid arthritis, psoriatic arthritis, or peripheral spondyloarthropathy may occur in association with Lyme disease.

Furthermore, lymphoid proliferation of salivary glands as a histopathologic sign of Sjögren's syndrome may be either benign or neoplastic, associated with non-Hodgkin lymphoma [9]. Mucosa-associated lymphoid tissue (MALT) lymphoma served in some studies as an in vivo model to evaluate complex interactions between B-lymphocytes and the microenvironment in the context of the neoplastic process. Infectious agents, such as Helicobacter pylori, Borrelia burgdorferi, Campylobacter jejuni, Chlamydia psittaci, hepatitis C virus as well as Epstein-Barr virus have been shown to be involved in MALT-lymphoma pathogenesis [10].

On the other hand, lymphadenopathy may be one of the early manifestations of Lyme disease caused by invasion of Borrelia burgdorferi into lymphatic nodes. The few existing experimental studies have confirmed that after infection with live Borrelia burgdorferi, the latter remains within lymphatic nodes, causing their rapid and significant enlargement, loss of demarcation between $B$ cell and $T$ cell zones, and exaggerated cytokine-mediated $B$ cell accumulation [11].

These interactions were the rationale for immunohistochemical analysis for exclusion of different lymphoma subtypes as well as for the search of infectious causes. Lyme borreliosis manifesting as Sjögren's syndrome and lymphadenopathy was confirmed by the performed laboratory studies and by the positive effect of antibacterial therapy. Taking into account the onset of Sjögren's syndrome after a tick bite and its symptomatic improvement after etiologic therapy for Lyme disease, we consider this to be a case of secondary Sjögren's syndrome, more than the primary type of Sjögren's syndrome.

\section{Conclusions}

This clinical case shows that patients with multisystem manifestations, particularly with lymphadenopathy and a history of an earlier tick bite, may also have a picture of Lyme disease. Such patients require a wider approach to diagnosis especially in histopathological interpretation of lymph node biopsy and treatment also directed at Borrelia burgdorferi infection.

The authors declare no conflict of interest.

\section{References}

1. Czupryna P, Moniuszko-Malinowska A, Pancewicz S, et al. Lyme disease in Poland - a serious problem? Adv Med Sci 2016; 61: 96-100.

2. Stricker RB, Fesler MC. Chronic Lyme disease: a working case definition. Am J Inf Dis 2018; 14: 1-44.

3. Malawista SE, Steere AC, Hardin JA. Lyme disease: a unique human model for an infectious etiology of rheumatic disease. Yale J Biol Med 1984; 57: 473-477.

4. Smiyan SI, Shkilna MI, Regalyuk VV, et al. Modern aspects of etiology, pathogenesis, clinical course and treatment of Lyme arthritis (review, own observations). Ukr J Rheumatol 2018; 72: 40-45.

5. Hinckley AF, Connally NP, Meek Jl, et al. Lyme disease testing by large commercial laboratories in the United States. Clin Infect Dis 2014; 59: 676-681.

6. Shiboski CH, Shiboski SC, Seror R, et al. International Sjögren's Syndrome Criteria Working Group. 2016 American College of Rheumatology/European League Against Rheumatism classification criteria for primary Sjögren's syndrome: a consensus and data-driven methodology involving three international patient cohorts. Ann Rheum Dis 2017; 76: 9-16.

7. Tunev SS, Hastey CJ, Hodzic E, et al. Lymphoadenopathy during Lyme borreliosis is caused by spirochete migration-induced specific B-cell activation. PLoS Pathog 2011; 7: e1002066.

8. Arvikar SL, Crowley JT, Sulka KB, Steere AC. Autoimmune arthritides, rheumatoid arthritis, psoriatic arthritis, or peripheral spondyloarthropathy, following Lyme disease. Arthritis Rheum 2017; 69: 194-202.

9. Alunno A, Leone MC, Giacomelli R, et al. Lymphoma and lymphomagenesis in primary Sjögren's syndrome. Front Med (Lausanne) 2018; 5: 102.

10. Guidoboni $M$, Ferreri AJ, Ponzoni $M$, et al. Infectious agents in mucosa-associated lymphoid tissue-type lymphomas: pathogenic role and therapeutic perspectives. Clin Lymphoma Myeloma 2006; 6: 289-300.

11. Oosting M, ter Hofstede $\mathrm{H}$, van de Veerdonk FL, et al. Role of interleukin-23 (IL-23) receptor signaling for IL-17 responses in human Lyme disease. Infect Immun 2011; 79: 4681-4687. 